\title{
An Approach to 1,1-Disubstituted Pyrazolylcyclopropane Building Blocks
}

\author{
Pavel S. Nosik ${ }^{\mathrm{a}, \mathrm{b}}$ \\ Oleksiy S. Artamonov ${ }^{a}$ \\ Sergey V. Ryabukhin*b \\ Oleksandr O. Grygorenkob \\ ${ }^{a}$ Institute of Organic Chemistry, National Academy of Sciences \\ of Ukraine, Murmanska Street 5, Kyiv 02660, Ukraine \\ s.v.ryabukhin@gmail.com \\ b Taras Shevchenko National University of Kyiv, Volodymyrska \\ Street 64, Kyiv 01601, Ukraine
}

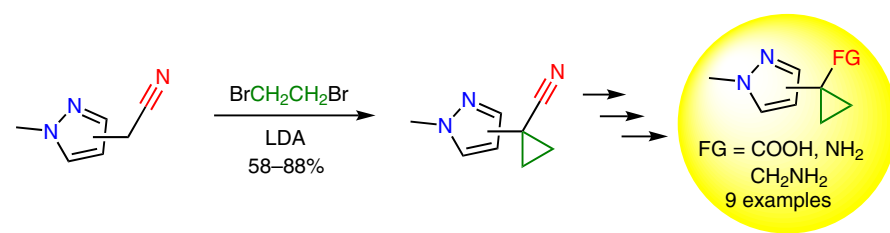

Received: 03.07.2017

Accepted after revision: 31.07.2017

Published online: 14.08 .2017

DOI: 10.1055/s-0036-1588544; Art ID: so-2017-d0029-op

License terms: CCO

Abstract An approach to isomeric 1,1-disubstituted pyrazolylcyclopropanes that relies on lithium diisopropylamide (LDA) mediated bisalkylation of the corresponding pyrazolylacetonitriles is developed. The building blocks obtained can be considered as lead-like bioisosteres of arylpyrazole and pyrazolecarboxamide moieties and are thus useful for early drug discovery projects.

Key words cyclopropanes, pyrazoles, hetarylacetonitriles, nucleophilic substitution, lead-oriented synthesis

1,1-Disubstituted (het)arylcyclopropanes are important chemotypes for the discovery of biologically active compounds. Arylcyclopropane derivatives are found among marketed drugs and drug candidates, including Lumacaftor 1, a drug for the treatment of cystic fibrosis approved by the FDA in 2015, ${ }^{1}$ CHF5074 or CSP-1103 (2), which has recently completed phase II clinical trials for Alzheimer's disease treatment, ${ }^{2}$ and MK-2894 (3), a potent and selective prostaglandin E2 subtype 4 receptor antagonist with potent antiinflammatory activity that is currently in preclinical studies $^{3}$ (Figure 1). The corresponding hetarylcyclopropanes have also demonstrated significant potential in medicinal chemistry, with derivatives showing nanomolar-range activity as GPR142 agonists $4\left(\mathrm{EC}_{50}=54 \mathrm{nM}\right){ }^{4}$ cannabinoid-1 (CB1) antagonists $5\left(\mathrm{IC}_{50}=0.7 \mathrm{nM}\right),{ }^{5}$ poly(ADP-ribose) polymerase (PARP) inhibitors $6\left(\mathrm{IC}_{50}=25 \mathrm{nM}\right),{ }^{6}$ and cathepsin $\mathrm{S}$ (Cat $\mathrm{S}$ ) inhibitors $7\left(\mathrm{IC}_{50}=0.5 \mathrm{nM}\right){ }^{7}$

The 1,1-disubstituted cyclopropane motif has been considered as a bioisosteric replacement of the double bond, amide carbonyl group, and ortho-disubstituted phenylene ring or its hetero-analogues (Figure 2). ${ }^{8}$ The latter idea was implemented into the design of highly potent inhibitors of Factor Xa (FXa). ${ }^{9}$

A general approach to the synthesis of 1,1-disubstituted (het)arylcyclopropanes relies on alkylation of (het)arylacetonitriles 8 with 1,2-dibromoethane or related bis-electro-<smiles>Cc1ccc(NC(=O)C2(c3ccc4c(c3)OC(F)(F)O4)CC2)nc1-c1cccc(C(=O)O)c1</smilesLumacaftor (1)<smiles>O=C(O)C1(c2ccc(-c3ccc(Cl)c(Cl)c3)c(F)c2)CC1</smiles>

CHF5074 or CSP-1103 (2)

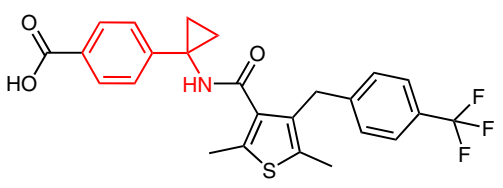

MK-2894 (3)<smiles>O=C(Nc1cc(-c2ccncc2)c[nH]c1=O)[C@H](Cc1ccccc1)NC(=O)C1(c2ccccn2)CC1</smiles>

GPR142 agonist 4 $\mathrm{EC}_{50}=54 \mathrm{nM}$

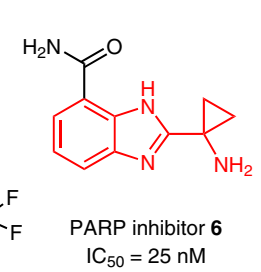

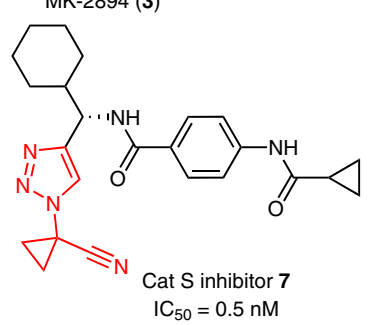

Figure 1 Biologically active 1,1-disubstituted (het)arylcyclopropanes 


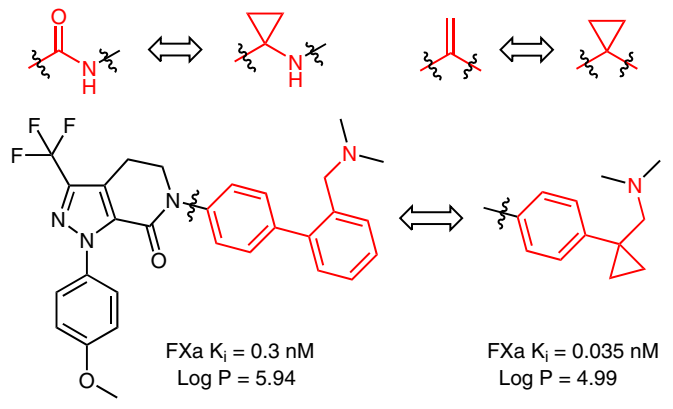

Figure 2 Bioisosteric replacements with 1,1-disubstituted cyclopropanes

philes in the presence of base (Scheme 1). The resulting 1(het)arylcyclopropanecarbonitriles $\mathbf{9}$ are versatile key intermediates for the preparation of other building blocks, for example, carboxylic acids and amines, as well as for the construction of heterocyclic rings. Most of the reports on the synthesis of compounds of general formula $\mathbf{9}$ are related to the substituted benzene derivatives. Several reports on the development of general procedures for the preparation of 1-arylcyclopropanecarbonitriles $\mathbf{9}(\mathrm{R}=\mathrm{Ar})$ from the corresponding arylacetonitriles $8(R=A r)$ have appeared; ${ }^{10,11}$ however, their hetaryl-substituted counterparts are far less studied in analogous transformations. A number of isolated examples that have been reported involve pyrrole, ${ }^{12}$ indole, ${ }^{10,13}$ thiophene, ${ }^{14}$ and pyridine ${ }^{15}$ derivatives. In some cases, it has been reported that arylation of cyclopropylcarbonitrile $\mathbf{1 0}^{16}$ or heterocyclizations involving 1-cyanocyclopropanecarboxylic acid or its derivatives $\mathbf{1 1}^{17}$ were more convenient for the preparation of 1-hetarylcyclopropanecarbonitriles $(\mathbf{9}, \mathrm{R}=\mathrm{Het} A r)$.

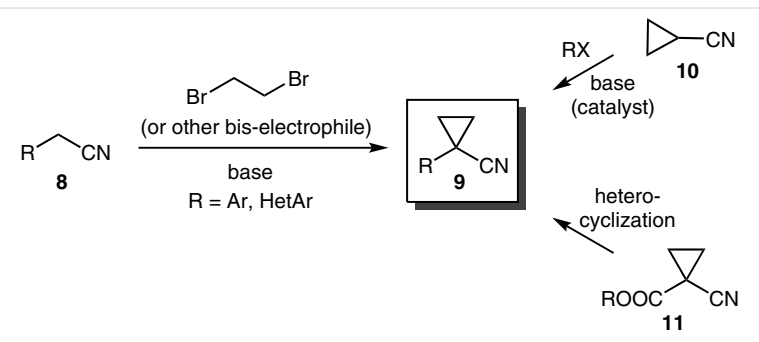

Scheme 1 Selected general approaches to the preparation of 1-(het)arylcyclopropanecarbonitriles 9

Recently, we ${ }^{18}$ and others ${ }^{19}$ have looked into the design and preparation of medicinal building blocks ${ }^{20}$ that are compatible with the concept of lead-oriented synthesis. ${ }^{21}$ As a part of this ongoing project, we became interested in the preparation of pyrazolyl-substituted building blocks 12-14 (Figure 3). Nitriles 9a-c are obvious key precursors for the preparation of these compounds. Since the corresponding pyrazolylacetonitriles 8a-c can be prepared from the known aldehydes $\mathbf{1 5 a}-\mathbf{c},{ }^{22}$ we turned to their double al- kylation as a possible method for the preparation of $\mathbf{9 a - c}$. It should be noted that, of the compounds $\mathbf{8 a}-\mathbf{c}$, only $\mathbf{8 b}$ was described previously. ${ }^{23}$ This was prepared by reaction of aldehyde 15b with TOSMIC in 65\% yield. For the preparation of $\mathbf{8 c}$, we used the same procedure, which gave the target product in $63 \%$ yield. In the case of $\mathbf{8 a}$, the method did not work well, and the target compound was formed in less than $20 \%$ yield; therefore, an alternative three-step route was used.

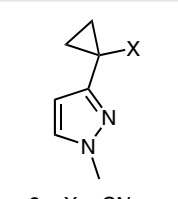

9a, $\mathrm{X}=\mathrm{CN}$

12a, $X=\mathrm{COOH}$

13a, $X=\mathrm{NH}_{2}$

14a, $X=\mathrm{CH}_{2} \mathrm{NH}_{2}$

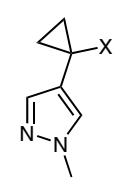

9b, $X=\mathrm{CN}$

12b, $X=\mathrm{COOH}$ 13b, $X=\mathrm{NH}_{2}$ 14b, $\mathrm{X}=\mathrm{CH}_{2} \mathrm{NH}_{2}$

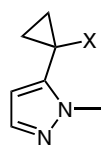

9c, $\mathrm{X}=\mathrm{CN}$

12c, $X=\mathrm{COOH}$ 13c, $\mathrm{X}=\mathrm{NH}_{2}$ 14c, $\mathrm{X}=\mathrm{CH}_{2} \mathrm{NH}_{2}$
Figure 3 1,1-Disubstituted pyrazolylcyclopropane building blocks and precursors for their synthesis

To our knowledge, no examples of bis-alkylation of hetarylacetonitriles $\mathbf{8}$ having a basic nitrogen atom with 1,2dibromoethane have been reported (the closest analogue contained an $\alpha$-chloropyridine moiety, ${ }^{15}$ which has low basicity). Typical conditions reported for alkylation of other substrates 8 with 1,2-dibromoethane are: (1) $\mathrm{NaOH}$ or $\mathrm{KOH}$, phase-transfer catalyst, toluene or $\mathrm{CH}_{2} \mathrm{Cl}_{2}-\mathrm{H}_{2} \mathrm{O}$, r.t. to reflux; (2) $\mathrm{NaH}$, DMF or DMSO, r.t. to $60^{\circ} \mathrm{C}$; (3) $t$-BuOK, DMSO, r.t. to $60{ }^{\circ} \mathrm{C}$; (4) KHMDS, THF, $-78{ }^{\circ} \mathrm{C}$ to r.t.

Surprisingly, these methods did not give satisfactory results for alkylation of the substrate $\mathbf{8 b}$ (Table 1, entries 1-6). Therefore, we turned to screening of other bases, and found that the use of LDA in THF led to the formation of the target product 9b in good yield (entry 8). Further experiments showed that the reaction could be performed without preliminary cooling to $-78{ }^{\circ} \mathrm{C}$. Hence, an operationally simple procedure was developed that allowed for the preparation of the target product in $69 \%$ yield (entry 9 ).

This simple and robust procedure for the transformation of $\mathbf{8}$ into $\mathbf{9}$ worked well with all three pyrazolylacetonitriles $\mathbf{8 a}-\mathbf{c}$, with the corresponding cyclopropanes $\mathbf{9 a}-\mathbf{c}$ being obtained in up to $10 \mathrm{~g}$ amounts and $57-82 \%$ yields. The versatility of these synthetic intermediates was demonstrated by their transformation into our initial target building blocks 12-14 (Scheme 2). In particular, alkaline hydrolysis of 9a-c gave carboxylic acids 12a-c in $94-99 \%$ yields (isolated as hydrochlorides). A modified Curtius reaction of 12a-c, followed by quenching of the intermediate isocyanate with tert-butanol, gave Boc derivatives 16a-c (51-64\% yields), which were transformed into amines 13a-c (isolated as dihydrochlorides) in nearly quantitative yields. Alternatively, 9a-c were reduced with borane-dimethylsulfoxide complex to give amines 14a-c (78-98\% yield). 
Table 1 Alkylation of the Substrates 8 b with 1,2-Dibromoethane under Various Reaction Conditions

\begin{tabular}{lll}
\hline Entry & Conditions & Yield of $\mathbf{9 b}(\%)^{\mathrm{a}}$ \\
\hline 1 & $\mathrm{NaOH}$, benzyl thiethylammonium chloride, r.t. & 0 \\
2 & $\mathrm{NaOH}$, tetrabutylammonium bromide, $50{ }^{\circ} \mathrm{C}$ & 0 \\
3 & $\mathrm{NaH}, \mathrm{DMF}$, r.t. & 0 \\
4 & $\mathrm{t}$-BuOK, DMSO, $60^{\circ} \mathrm{C}$ & 0 \\
5 & $\mathrm{KHMDS}, \mathrm{THF},-78{ }^{\circ} \mathrm{C}$ & 0 \\
6 & $\mathrm{KHMDS}, \mathrm{THF}, 0^{\circ} \mathrm{C}$ to r.t. & 40 \\
7 & $\mathrm{LDA}, \mathrm{THF},-78{ }^{\circ} \mathrm{C}$ & 31 \\
8 & $\mathrm{LDA}, \mathrm{THF},-78{ }^{\circ} \mathrm{C}$ to r.t. & $70^{\mathrm{b}}$ \\
9 & $\mathrm{LDA}, \mathrm{THF}, 0{ }^{\circ} \mathrm{C}$ to r.t. & $69^{\mathrm{b}}$ \\
\hline
\end{tabular}

${ }^{a}$ Unless noted otherwise, yield was based on LCMS or ${ }^{1} \mathrm{H}$ NMR analysis. ${ }^{\mathrm{b}}$ Isolated yield.

$$
\text { }
$$$$
{ }^{n} \overbrace{}^{1}
$$$$
57-82 \% \mid \text { LDA }
$$<smiles>Cn1nccc1C1(C2(C)CC2)CC1</smiles>
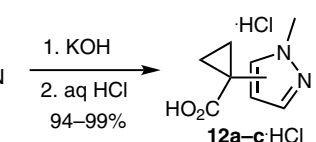

8a, 9a, 12a-16a: pyrazol-3-yl 8b, 9b, 12b-16b: pyrazol-4-yl 8c, 9c, 12c-16c: pyrazol-5-yl
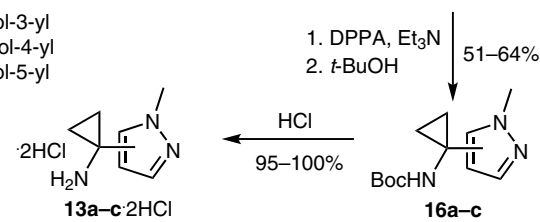

Scheme 2 Synthesis of pyrazolylcyclopropane building blocks 12-14

In conclusion, a convenient approach to 1,1-disubstituted pyrazolylcyclopropane building blocks has been developed that is amendable for multigram-scale preparation. These products are promising building blocks for lead-oriented synthesis in medicinal chemistry, in particular as lead-like bioisosteric replacements of arylpyrazole or pyrazolecarboxamide moieties. They are low-molecular-weight (MW $=137-166$ ), hydrophilic ( $\log \mathrm{P}=-0.16$ to 1.06 ), have a limited number of polar atoms and rotatable bonds, and a reasonable fraction of $\mathrm{sp}^{3}$ carbon atoms $\left(\mathrm{Fsp}^{3}=0.50-0.63\right.$ ) (Table 2). ${ }^{24}$ Moreover, the pyrazolylcyclopropane scaffold complies with the 'biocore' concept for the scaffold design proposed by Kombarov and co-workers. ${ }^{25}$ The procedure described can also be useful for the preparation of other 1,1-disubstituted (het)arylcyclopropanes, especially those containing basic nitrogen atoms.
Table 2 Calculated Physicochemical Parameters of the Building Blocks 12-14 Prepared in this Work ${ }^{\mathrm{a}}$

\begin{tabular}{lccccccc}
\hline Compound & MW & cLog P & HAcc & HDon & RotB & TPSA $\left(\AA^{2}\right)$ & Fsp $^{3}$ \\
\hline 12a & 166 & 1.06 & 3 & 1 & 2 & 55.1 & 0.50 \\
12b & 166 & 0.56 & 3 & 1 & 2 & 55.1 & 0.50 \\
12c & 166 & 0.47 & 3 & 1 & 2 & 55.1 & 0.50 \\
13a & 137 & 0.30 & 2 & 1 & 1 & 43.8 & 0.57 \\
13b & 137 & -0.08 & 2 & 1 & 1 & 43.8 & 0.57 \\
13c & 137 & -0.16 & 2 & 1 & 1 & 43.8 & 0.57 \\
14a & 151 & 0.48 & 2 & 1 & 2 & 43.8 & 0.63 \\
14b & 151 & 0.09 & 2 & 1 & 2 & 43.8 & 0.63 \\
14c & 151 & 0.01 & 2 & 1 & 2 & 43.8 & 0.63 \\
\hline
\end{tabular}

a MW: molecular weight; cLog P: calculated partitioning coefficient logarithm; HAcc: hydrogen-bond acceptor count; HDon: hydrogen-bond donor count; RotB: number of rotatable bonds; TPSA: total polar surface area; $\mathrm{Fsp}^{3}$ : fraction of $\mathrm{sp}^{3}$ carbon atoms.

The solvents were purified according to standard procedures. ${ }^{26}$ Compounds $\mathbf{1 5 a},{ }^{22 a} \mathbf{8 b},{ }^{23}$ and $\mathbf{1 5} \mathbf{c}^{22 b}$ were prepared by using reported methods. All other starting materials were purchased from commercial sources. Analytical TLC was performed using Polychrom SI F254 plates. Column chromatography was performed using Kieselgel Merck $60(230-400 \mathrm{mesh})$ as the stationary phase. ${ }^{1} \mathrm{H}$ and ${ }^{13} \mathrm{C}$ NMR spectra were recorded with a Varian Gemini 2000 spectrometer (at $400 \mathrm{MHz}$ for ${ }^{1} \mathrm{H}$ and $101 \mathrm{MHz}$ for ${ }^{13} \mathrm{C}$ NMR). Chemical shifts are reported in ppm downfield from TMS $\left({ }^{1} \mathrm{H},{ }^{13} \mathrm{C}\right)$ as an internal standard. Mass spectra were recorded with an Agilent 1100 LCMSD SL instrument (electrospray ionization (APESI)).

\section{(1-Methyl-1H-pyrazol-3-yl)methanol (17)}

To a solution of aldehyde 15a (58.3 g, $0.529 \mathrm{~mol})$ in $\mathrm{MeOH}(750 \mathrm{~mL})$, $\mathrm{NaBH}_{4}(40.0 \mathrm{~g}, 1.06 \mathrm{~mol})$ was added portionwise at $0{ }^{\circ} \mathrm{C}$. The resulting mixture was stirred overnight at r.t., then evaporated, diluted with $10 \%$ aq $\mathrm{NaOH}(500 \mathrm{~mL})$, and extracted with EtOAc $(3 \times 250 \mathrm{~mL})$. The combined organic layers were dried over $\mathrm{Na}_{2} \mathrm{SO}_{4}$, filtered, and evaporated in vacuo to give $\mathbf{1 7}$, which was pure enough for the next step. An analytically pure sample was obtained by vacuum distillation.

Yield: $49.5 \mathrm{~g}$ (84\%); colorless oil; bp $70-72{ }^{\circ} \mathrm{C} / 1$ mbar.

${ }^{1} \mathrm{H}$ NMR $\left(\mathrm{CDCl}_{3}, 400 \mathrm{MHz}\right): \delta=7.28(\mathrm{~d}, J=2.2 \mathrm{~Hz}, 1 \mathrm{H}), 6.22(\mathrm{~d}, J=$ $2.2 \mathrm{~Hz}, 1 \mathrm{H}$ ), 4.79 (br. s, $1 \mathrm{H}$ ), 4.64 (s, $2 \mathrm{H}$ ), 3.85 (s, $3 \mathrm{H}$ ).

${ }^{13} \mathrm{C} \mathrm{NMR}\left(\mathrm{CDCl}_{3}, 101 \mathrm{MHz}\right): \delta=152.0,130.6,104.0,57.9,38.3$.

MS (ESI): $m / z=113\left[\mathrm{MH}^{+}\right]$.

Anal. Calcd for $\mathrm{C}_{5} \mathrm{H}_{8} \mathrm{~N}_{2} \mathrm{O}: \mathrm{C}, 53.56 ; \mathrm{H}, 7.19 ; \mathrm{N}, 24.98$. Found: C, 53.47; H, 6.84; N, 24.75 .

\section{3-(Chloromethyl)-1-methyl-1H-pyrazole Hydrochloride (18)}

To a solution of $\mathbf{1 7}(12.0 \mathrm{~g}, 0.107 \mathrm{~mol})$ in anhydrous $\mathrm{CH}_{2} \mathrm{Cl}_{2}(150 \mathrm{~mL})$, $\mathrm{SOCl}_{2}(19.1 \mathrm{~g}, 0.16 \mathrm{~mol})$ was added dropwise at $0{ }^{\circ} \mathrm{C}$. The reaction mixture was stirred overnight at r.t., then evaporated in vacuo, and the solid was triturated with anhydrous $\mathrm{Et}_{2} \mathrm{O}(200 \mathrm{~mL})$, filtered, washed with anhydrous $\mathrm{Et}_{2} \mathrm{O}(100 \mathrm{~mL})$, and dried under reduced pressure to give 18. An analytically pure sample was obtained by recrystallization from acetone.

Yield: $17.0 \mathrm{~g}$ (95\%); white solid; $\mathrm{mp} 136-138^{\circ} \mathrm{C}$. 
${ }^{1} \mathrm{H}$ NMR $\left(400 \mathrm{MHz}\right.$, DMSO- $\left.d_{6}\right): \delta=9.86$ (br. s, $\left.1 \mathrm{H}\right), 7.64(\mathrm{~d}, J=2.1 \mathrm{~Hz}$, $1 \mathrm{H}), 6.27$ (d, J = 2.1 Hz, $1 \mathrm{H}), 4.64(\mathrm{~s}, 2 \mathrm{H}), 3.79(\mathrm{~s}, 3 \mathrm{H})$.

${ }^{13} \mathrm{C}$ NMR (101 MHz, DMSO- $\left.d_{6}\right): \delta=147.4,131.9,105.2,39.2,38.4$.

MS (ESI): $m / z=131 / 133\left[\mathrm{MH}^{+}\right]$.

Anal. Calcd for $\mathrm{C}_{5} \mathrm{H}_{8} \mathrm{Cl}_{2} \mathrm{~N}_{2}$ : C, 35.95; $\mathrm{H}, 4.83 ; \mathrm{N}, 16.77 ; \mathrm{Cl}, 42.45$. Found: C, 35.91; H, 4.72; N, 16.81; Cl, 42.70.

\section{2-(1-Methyl-1H-pyrazol-3-yl)acetonitrile (8a)}

Compound 18 (9.70 g, $58.1 \mathrm{mmol}$ ) was dissolved in DMSO (300 mL), $\mathrm{KCN}(15.1 \mathrm{~g}, 0.232 \mathrm{~mol})$ was added, and the mixture was stirred at r.t. overnight, then diluted with $\mathrm{H}_{2} \mathrm{O}(600 \mathrm{~mL})$, and extracted with EtOAc $(3 \times 250 \mathrm{~mL})$. The combined extracts were washed with brine $(250$ $\mathrm{mL}$ ), dried over $\mathrm{Na}_{2} \mathrm{SO}_{4}$, filtered, and concentrated in vacuo to give $8 \mathbf{a}$. An analytically pure sample was obtained by column chromatography (EtOAc-hexanes, 1:2).

Yield: $6.51 \mathrm{~g}$ (92\%); reddish oil; $R_{f} 0.62$ (EtOAc-hexanes, $1: 2$ ).

${ }^{1} \mathrm{H}$ NMR $\left(400 \mathrm{MHz}, \mathrm{CDCl}_{3}\right): \delta=7.30(\mathrm{~d}, J=2.2 \mathrm{~Hz}, 1 \mathrm{H}), 6.20(\mathrm{~d}, J=$ $2.2 \mathrm{~Hz}, 1 \mathrm{H}), 3.82(\mathrm{~s}, 3 \mathrm{H}), 3.69$ (s, $2 \mathrm{H}$ ).

${ }^{13} \mathrm{C}$ NMR $\left(101 \mathrm{MHz}, \mathrm{CDCl}_{3}\right): \delta=140.8,131.3,116.9,104.5,38.6,17.2$.

MS (ESI): $m / z=122\left[\mathrm{MH}^{+}\right]$.

Anal. Calcd for $\mathrm{C}_{6} \mathrm{H}_{7} \mathrm{~N}_{3}$ : C, 59.49; $\mathrm{H}$ 5.82; N, 34.69. Found: C, 59.49; $\mathrm{H}$ 5.82; N, 34.69.

\section{2-(1-Methyl-1H-pyrazol-5-yl)acetonitrile (8c)}

Potassium tert-butoxide ( $23.5 \mathrm{~g}, 95 \%, 0.199 \mathrm{~mol})$ was suspended in anhydrous 1,2-dimethoxyethane $(90 \mathrm{~mL})$, and the mixture was cooled to $-60{ }^{\circ} \mathrm{C}$. TosMIC ( $23.8 \mathrm{~g}, 0.122 \mathrm{~mol}$ ) was dissolved in anhydrous $1,2-$ dimethoxyethane $(75 \mathrm{~mL})$, and the resulting solution was added dropwise to the potassium tert-butoxide solution over $20 \mathrm{~min}$. After stirring for $20 \mathrm{~min}$ at -60 to $-55^{\circ} \mathrm{C}$, aldehyde $15 \mathrm{c}(9.00 \mathrm{~g}, 0.0816 \mathrm{~mol})$ in anhydrous 1,2-dimethoxyethane $(55 \mathrm{~mL})$ was added over $23 \mathrm{~min}$. The mixture was stirred at -55 to $-50^{\circ} \mathrm{C}$ for $1 \mathrm{~h}$ to yield a thick suspension. $\mathrm{MeOH}(90 \mathrm{~mL})$ was then added, which gave a clear brown solution. The cooling bath was removed and, after stirring at r.t. for 5 min, the reaction flask was placed into an oil bath preheated to $85^{\circ} \mathrm{C}$. The reaction mixture was stirred for $1 \mathrm{~h}$, then cooled and concentrated in vacuo. The residue was dissolved in $\mathrm{H}_{2} \mathrm{O}(180 \mathrm{~mL})$ and $\mathrm{AcOH}(9$ $\mathrm{mL})$. The mixture was extracted with EtOAc $(3 \times 250 \mathrm{~mL})$, and the combined extracts were washed with brine $(100 \mathrm{~mL})$, dried over $\mathrm{Na}_{2} \mathrm{SO}_{4}$, filtered, and concentrated in vacuo to give a brown oil. The crude product was distilled under reduced pressure to give $\mathbf{8 c}$.

Yield: $6.22 \mathrm{~g}$ (63\%); reddish oil; bp $65-66{ }^{\circ} \mathrm{C} / 0.1 \mathrm{mbar}$.

${ }^{1} \mathrm{H}$ NMR $\left(400 \mathrm{MHz}, \mathrm{CDCl}_{3}\right): \delta=7.37(\mathrm{~d}, J=1.9 \mathrm{~Hz}, 1 \mathrm{H}), 6.22(\mathrm{~d}, J=$ $1.9 \mathrm{~Hz}, 1 \mathrm{H}), 3.80$ (s, $3 \mathrm{H}), 3.72(\mathrm{~s}, 2 \mathrm{H})$.

${ }^{13} \mathrm{C}$ NMR $\left(101 \mathrm{MHz}, \mathrm{CDCl}_{3}\right): \delta=138.2,130.2,114.8,106.3,36.3,15.1$. MS (ESI): $m / z=122\left[\mathrm{MH}^{+}\right]$.

Anal. Calcd for $\mathrm{C}_{6} \mathrm{H}_{7} \mathrm{~N}_{3}$ : C, 59.49; $\mathrm{H}$ 5.82; N, 34.69. Found: C, 59.61; $\mathrm{H}$, $6.18 ; \mathrm{N}, 34.77$.

\section{Preparation of Nitriles 9; General Procedure}

To a solution of diisopropylamine ( $30.4 \mathrm{~g}, 0.300 \mathrm{~mol}$ ) in THF (150 mL), $n$-BuLi (78.8 mL, $0.260 \mathrm{~mol}, 3.3 \mathrm{M}$ in hexanes) was added at $-30{ }^{\circ} \mathrm{C}$, and the mixture was stirred at r.t. for $1 \mathrm{~h}$. Nitrile $8 \mathbf{a}-\mathbf{c}(12.1 \mathrm{~g}, 0.100$ $\mathrm{mol})$ in THF $(50 \mathrm{~mL})$ was added at $0{ }^{\circ} \mathrm{C}$, and the resulting mixture was stirred at r.t. for 1 h. 1,2-Dibromoethane $(9.48 \mathrm{~mL}, 0.110 \mathrm{~mol})$ was added at $0{ }^{\circ} \mathrm{C}$, and the reaction mixture was stirred for an additional 1 $\mathrm{h}$, then quenched with saturated aq $\mathrm{NH}_{4} \mathrm{Cl}(100 \mathrm{~mL})$ and extracted with $\mathrm{CH}_{2} \mathrm{Cl}_{2}(2 \times 100 \mathrm{~mL})$. The combined organic extracts were dried over $\mathrm{Na}_{2} \mathrm{SO}_{4}$, filtered, and evaporated in vacuo. The crude product was purified by column chromatography (hexanes-EtOAc, 4:1).

\section{1-(1-Methyl-1H-pyrazol-3-yl)cyclopropanecarbonitrile (9a)}

Yield: $5.62 \mathrm{~g}$ (57\%); reddish oil; $R_{f} 0.70$ (hexanes-EtOAc, 4:1).

${ }^{1} \mathrm{H}$ NMR $\left(400 \mathrm{MHz}, \mathrm{CDCl}_{3}\right): \delta=7.26(\mathrm{~d}, J=2.2 \mathrm{~Hz}, 1 \mathrm{H}), 6.24(\mathrm{~d}, J=$ $2.2 \mathrm{~Hz}, 1 \mathrm{H}), 3.81$ (s, $3 \mathrm{H}), 1.65-1.59(\mathrm{~m}, 2 \mathrm{H}), 1.52-1.47$ (m, $2 \mathrm{H})$.

${ }^{13} \mathrm{C}$ NMR $\left(101 \mathrm{MHz}, \mathrm{CDCl}_{3}\right): \delta=147.3,130.8,121.9,103.3,38.7,17.8$, 9.0.

MS (ESI): $m / z=148\left[\mathrm{MH}^{+}\right], 121\left[\mathrm{M}^{+}-\mathrm{CN}\right]$.

Anal. Calcd for $\mathrm{C}_{8} \mathrm{H}_{9} \mathrm{~N}_{3}$ : C, 65.29; $\mathrm{H}, 6.16 ; \mathrm{N}, 28.55$. Found: C, 65.07; $\mathrm{H}$, 5.79; N, 28.67.

\section{1-(1-Methyl-1H-pyrazol-4-yl)cyclopropanecarbonitrile (9b)}

Yield: $6.80 \mathrm{~g}$ (69\%); yellowish oil; $R_{f} 0.68$ (hexanes-EtOAc, 4:1).

${ }^{1} \mathrm{H} \mathrm{NMR}\left(400 \mathrm{MHz}, \mathrm{CDCl}_{3}\right): \delta=7.37(\mathrm{~s}, 1 \mathrm{H}), 7.31(\mathrm{~s}, 1 \mathrm{H}), 3.86(\mathrm{~s}, 3 \mathrm{H})$, $1.64-1.59(\mathrm{~m}, 2 \mathrm{H}), 1.24-1.20(\mathrm{~m}, 2 \mathrm{H})$.

${ }^{13} \mathrm{C}$ NMR $\left(101 \mathrm{MHz}, \mathrm{CDCl}_{3}\right): \delta=136.6,128.7,122.6,118.5,39.3,17.8$, 5.6.

MS (ESI): $m / z=148\left[\mathrm{MH}^{+}\right]$.

Anal. Calcd for $\mathrm{C}_{8} \mathrm{H}_{9} \mathrm{~N}_{3}: \mathrm{C}, 65.29 ; \mathrm{H}, 6.16 ; \mathrm{N}, 28.55$. Found: C, 65.31; $\mathrm{H}$, $6.12 ; \mathrm{N}, 28.45$.

1-(1-Methyl-1H-pyrazol-5-yl)cyclopropanecarbonitrile (9c)

Yield: $8.09 \mathrm{~g}$ (82\%); colorless oil; $R_{f} 0.73$ (hexanes-EtOAc, 4:1).

${ }^{1} \mathrm{H} \mathrm{NMR}\left(400 \mathrm{MHz}, \mathrm{CDCl}_{3}\right): \delta=7.35(\mathrm{~d}, J=1.9 \mathrm{~Hz}, 1 \mathrm{H}), 6.07(\mathrm{~d}, J=$ $1.9 \mathrm{~Hz}, 1 \mathrm{H}), 3.99(\mathrm{~s}, 3 \mathrm{H}), 1.70(\mathrm{dd}, J=7.6,4.9 \mathrm{~Hz}, 2 \mathrm{H}), 1.34(\mathrm{dd}, J=$ 7.6, $4.9 \mathrm{~Hz}, 2 \mathrm{H})$.

${ }^{13} \mathrm{C}$ NMR $\left(101 \mathrm{MHz}, \mathrm{CDCl}_{3}\right): \delta=137.8,136.5,119.9,105.9,36.8,15.9$, 5.0.

MS (ESI): $m / z=148\left[\mathrm{MH}^{+}\right], 121\left[\mathrm{M}^{+}-\mathrm{CN}\right]$.

Anal. Calcd for $\mathrm{C}_{8} \mathrm{H}_{9} \mathrm{~N}_{3}: \mathrm{C}, 65.29 ; \mathrm{H}, 6.16 ; \mathrm{N}, 28.55$. Found: C, 65.48; $\mathrm{H}$, $6.33 ; \mathrm{N}, 28.39$.

\section{Preparation of Carboxylic Acids 12; General Procedure}

A suspension of nitrile $\mathbf{9}$ ( $3.50 \mathrm{~g}, 0.0238 \mathrm{~mol})$ in $10 \%$ aq $\mathrm{NaOH}(100$ $\mathrm{mL}$ ) was heated at reflux overnight (until $\mathrm{NH}_{3}$ evolution ceased). The homogeneous solution was washed with $\mathrm{Et}_{2} \mathrm{O}(50 \mathrm{~mL})$, the aqueous layer was acidified with $6 \mathrm{M}$ aq $\mathrm{HCl}$ to $\mathrm{pH} 3$, and evaporated to dryness. The residue was triturated with $i-\operatorname{PrOH}(100 \mathrm{~mL})$, the solid was filtered off, washed with $i$-PrOH $(50 \mathrm{~mL})$, and the combined filtrates were evaporated in vacuo. The residue was triturated with acetone $(50 \mathrm{~mL})$, filtered, and dried under reduced pressure to give 12. An analytically pure sample was obtained by recrystallization from acetone-EtOH.

\section{1-(1-Methyl-1H-pyrazol-3-yl)cyclopropanecarboxylic Acid (12a)}

Yield: $4.62 \mathrm{~g}$ (96\%); white solid; $\mathrm{mp} 182-183^{\circ} \mathrm{C}$.

${ }^{1} \mathrm{H}$ NMR $\left(400 \mathrm{MHz}\right.$, DMSO- $\left.d_{6}\right): \delta=7.60(\mathrm{~d}, J=1.8 \mathrm{~Hz}, 1 \mathrm{H}), 6.32(\mathrm{~d}, J=$ $2.0 \mathrm{~Hz}, 1 \mathrm{H}), 3.77(\mathrm{~s}, 3 \mathrm{H}), 1.41$ (dd, $J=6.7,3.4 \mathrm{~Hz}, 2 \mathrm{H}), 1.24$ (dd, $J=$ 6.7, $3.5 \mathrm{~Hz}, 2 \mathrm{H}), \mathrm{COOH}$ is exchanged with $\mathrm{HDO}$.

${ }^{13} \mathrm{C}$ NMR (101 MHz, DMSO- $\left.d_{6}\right): \delta=174.2,149.3,131.3,105.8,38.2$, 21.9, 17.1.

MS (ESI): $m / z=167\left[\mathrm{MH}^{+}\right], 149\left[\mathrm{M}^{+}-\mathrm{OH}\right], 121\left[\mathrm{M}^{+}-\mathrm{COOH}\right]$. 
Anal. Calcd for $\mathrm{C}_{8} \mathrm{H}_{10} \mathrm{~N}_{2} \mathrm{O}_{2}$ : C, 57.82; $\mathrm{H}, 6.07 ; \mathrm{N}, 16.86$. Found: C, 58.20; $\mathrm{H}, 5.81 ; \mathrm{N}, 17.20$.

\section{1-(1-Methyl-1H-pyrazol-4-yl)cyclopropanecarboxylic Acid (12b)}

Yield: $4.50 \mathrm{~g}(94 \%)$; white solid; mp 168-170 ${ }^{\circ} \mathrm{C}$.

${ }^{1} \mathrm{H}$ NMR $\left(400 \mathrm{MHz}\right.$, DMSO- $\left.d_{6}\right): \delta=7.72(\mathrm{~s}, 1 \mathrm{H}), 7.43(\mathrm{~s}, 1 \mathrm{H}), 3.79(\mathrm{~s}$, $3 \mathrm{H}), 1.48-1.39(\mathrm{~m}, 2 \mathrm{H}), 1.10-1.05(\mathrm{~m}, 2 \mathrm{H}), \mathrm{COOH}$ is exchanged with HDO.

${ }^{13} \mathrm{C}$ NMR (101 MHz, DMSO- $\left.d_{6}\right): \delta=175.4,138.1,130.9,121.0,38.7$, 19.4, 18.1.

MS (ESI): $m / z=167\left[\mathrm{MH}^{+}\right], 121\left[\mathrm{M}^{+}-\mathrm{COOH}\right]$.

Anal. Calcd for $\mathrm{C}_{8} \mathrm{H}_{10} \mathrm{~N}_{2} \mathrm{O}_{2}$ : C, 57.82; $\mathrm{H}, 6.07 ; \mathrm{N}, 16.86$. Found: C, 57.83; $\mathrm{H}, 5.8 ; \mathrm{N}, 16.95$.

\section{1-(1-Methyl-1H-pyrazol-5-yl)cyclopropanecarboxylic Acid (12c)}

Yield: $4.81 \mathrm{~g}$ (99\%); white solid; mp 177-179 ${ }^{\circ} \mathrm{C}$.

${ }^{1} \mathrm{H}$ NMR (400 MHz, DMSO- $d_{6}$ ): $\delta=7.42(\mathrm{~s}, 1 \mathrm{H}), 6.20(\mathrm{~s}, 1 \mathrm{H}), 3.77(\mathrm{~s}$, $3 \mathrm{H}), 1.53(\mathrm{dd}, J=7.0,3.9 \mathrm{~Hz}, 2 \mathrm{H}), 1.23(\mathrm{dd}, J=7.1,3.9 \mathrm{~Hz}, 2 \mathrm{H}), \mathrm{COOH}$ is exchanged with HDO.

${ }^{13} \mathrm{C}$ NMR $\left(101 \mathrm{MHz}\right.$, DMSO- $\left.d_{6}\right): \delta=173.1,141.6,136.4,106.3,36.3$, 19.3, 16.2 .

MS (ESI): $m / z=167\left[\mathrm{MH}^{+}\right], 121\left[\mathrm{M}^{+}-\mathrm{COOH}\right]$.

Anal. Calcd for $\mathrm{C}_{8} \mathrm{H}_{10} \mathrm{~N}_{2} \mathrm{O}_{2}$ : C, 57.82; $\mathrm{H}, 6.07 ; \mathrm{N}, 16.86$. Found: C, 58.08; $\mathrm{H}, 6.18 ; \mathrm{N}, 16.53$.

\section{Preparation of 16; General Procedure}

To a solution of carboxylic acid $12(1.60 \mathrm{~g}, 9.58 \mathrm{mmol})$ in toluene (20 $\mathrm{mL}$ ), $\mathrm{NEt}_{3}(2.96 \mathrm{~mL}, 0.0213 \mathrm{~mol}$ ) was added, followed by DPPA (3.25 g, $0.0118 \mathrm{~mol})$ in toluene $(5 \mathrm{~mL})$ dropwise at $40{ }^{\circ} \mathrm{C}$. The resulting mixture was heated at reflux for $3 \mathrm{~h}$. tert-Butanol $(5.90 \mathrm{~g}, 0.0797 \mathrm{~mol})$ was added, and the resulting mixture was heated at reflux overnight. The solvent was evaporated and the residue was dissolved in EtOAc $(50 \mathrm{~mL})$, washed with $\mathrm{H}_{2} \mathrm{O}(20 \mathrm{~mL}), 15 \%$ aq citric acid $(20 \mathrm{~mL})$, and saturated aq $\mathrm{NaHCO}_{3}(20 \mathrm{~mL})$. The organic layer was dried over $\mathrm{Na}_{2} \mathrm{SO}_{4}$, filtered, and evaporated in vacuo. The crude product was purified by column chromatography (EtOAc-hexanes, 1:1).

\section{tert-Butyl 1-(1-Methyl-1H-pyrazol-3-yl)cyclopropylcarbamate} (16a)

Yield: $0.953 \mathrm{~g}$ (51\%); yellowish oil; $R_{f} 0.43$ (EtOAc-hexanes, $1: 1$ ). ${ }^{1} \mathrm{H} \mathrm{NMR}\left(400 \mathrm{MHz}, \mathrm{CDCl}_{3}\right): \delta=7.21(\mathrm{~d}, J=2.2 \mathrm{~Hz}, 1 \mathrm{H}), 6.10$ (br. s, $\left.1 \mathrm{H}\right)$, 5.34 (br. s, $1 \mathrm{H}), 3.80$ (s, $3 \mathrm{H}), 1.45$ (s, $9 \mathrm{H}), 1.33-1.26$ (m, $2 \mathrm{H}), 1.21-$ $1.14(\mathrm{~m}, 2 \mathrm{H})$.

${ }^{13} \mathrm{C}$ NMR (101 MHz, $\mathrm{CDCl}_{3}$ ): $\delta$ (two rotamers) $=155.4$ and 154.6, 130.3, 129.3 and 124.7, 102.4, 79.0, 38.4, 30.9 and 29.4, 28.1, 17.2.

MS (ESI): $m / z=260\left[\mathrm{MNa}^{+}\right], 238\left[\mathrm{MH}^{+}\right], 182\left[\mathrm{MH}^{+}-\mathrm{C}_{4} \mathrm{H}_{9}\right], 138$ $\left[\mathrm{MH}^{+}-\mathrm{CO}_{2}-\mathrm{C}_{4} \mathrm{H}_{8}\right]$.

Anal. Calcd for $\mathrm{C}_{12} \mathrm{H}_{19} \mathrm{~N}_{3} \mathrm{O}_{2}$ : C, 60.74; H, 8.07; N, 17.71. Found: $\mathrm{C}$, $60.82 ; \mathrm{H}, 7.81 ; \mathrm{N}, 17.55$.

\section{tert-Butyl 1-(1-Methyl-1H-pyrazol-4-yl)cyclopropylcarbamate (16b)}

Yield: $1.10 \mathrm{~g}$ (59\%); white solid; mp $112-114^{\circ} \mathrm{C} ; R_{f} 0.38$ (EtOAchexanes, 1:1).

${ }^{1} \mathrm{H}$ NMR (400 MHz, $\left.\mathrm{CDCl}_{3}\right): \delta=7.28(\mathrm{~s}, 1 \mathrm{H}), 7.27(\mathrm{~s}, 1 \mathrm{H}), 5.23(\mathrm{~s}, 1 \mathrm{H})$, $3.83(\mathrm{~s}, 3 \mathrm{H}), 1.44(\mathrm{~s}, 9 \mathrm{H}), 1.17-1.11(\mathrm{~m}, 2 \mathrm{H}), 1.04-0.97(\mathrm{~m}, 2 \mathrm{H})$
${ }^{13} \mathrm{C}$ NMR $\left(101 \mathrm{MHz}, \mathrm{CDCl}_{3}\right)$ : $\delta$ (two rotamers) $=156.6$ and 155.6, $136.8,128.7$ and $127.9,125.8$ and $124.1,79.4,38.8,28.3,16.9,16.3$.

MS (ESI): $m / z=238\left[\mathrm{MH}^{+}\right], 182\left[\mathrm{MH}^{+}-\mathrm{C}_{4} \mathrm{H}_{9}\right]$.

Anal. Calcd for $\mathrm{C}_{12} \mathrm{H}_{19} \mathrm{~N}_{3} \mathrm{O}_{2}$ : C, 60.74; $\mathrm{H}, 8.07 ; \mathrm{N}, 17.71$. Found: C, $60.73 ; \mathrm{H}, 8.47 ; \mathrm{N}, 17.48$.

\section{tert-Butyl 1-(1-Methyl-1H-pyrazol-5-yl)cyclopropylcarbamate} (16c)

Yield: $1.17 \mathrm{~g}$ (64\%); white solid; $\mathrm{mp} 97-99^{\circ} \mathrm{C} ; R_{f} 0.40$ (EtOAchexanes, $1: 1)$.

${ }^{1} \mathrm{H} \mathrm{NMR}\left(400 \mathrm{MHz}, \mathrm{CDCl}_{3}\right): \delta=7.33(\mathrm{~d}, J=0.9 \mathrm{~Hz}, 1 \mathrm{H}), 6.18$ (br. s, $1 \mathrm{H}$ ), 5.24 (br s, $1 \mathrm{H}), 4.00$ (s, $3 \mathrm{H}), 1.39$ (s, $9 \mathrm{H}), 1.31-1.21$ (m, $2 \mathrm{H}), 1.20$ $1.05(\mathrm{~m}, 2 \mathrm{H})$.

${ }^{13} \mathrm{C}$ NMR $\left(101 \mathrm{MHz}, \mathrm{CDCl}_{3}\right): \delta=154.4,143.0,137.4,106.0,79.4,36.9$, 28.0, 26.9, 15.0 .

MS (ESI): $m / z=238\left[\mathrm{MH}^{+}\right], 182\left[\mathrm{MH}^{+}-\mathrm{C}_{4} \mathrm{H}_{9}\right], 138\left[\mathrm{MH}^{+}-\mathrm{CO}_{2}-\mathrm{C}_{4} \mathrm{H}_{8}\right]$, $121\left[\mathrm{M}^{+}-\mathrm{NHBoc}\right]$.

Anal. Calcd for $\mathrm{C}_{12} \mathrm{H}_{19} \mathrm{~N}_{3} \mathrm{O}_{2}$ : C, 60.74; $\mathrm{H}, 8.07 ; \mathrm{N}, 17.71$. Found: C, 61.14; H, 7.89; N, 17.91.

\section{Preparation of 13.2HCl; General Procedure}

To a solution of $\mathbf{1 6}(0.500 \mathrm{~g}, 2.11 \mathrm{mmol})$ in $\mathrm{Et}_{2} \mathrm{O}(10 \mathrm{~mL})$, saturated $\mathrm{HCl}$ in $\mathrm{Et}_{2} \mathrm{O}(20 \mathrm{~mL})$ was added, and the reaction mixture was stirred at r.t. overnight. The precipitate was filtered, washed with $\mathrm{Et}_{2} \mathrm{O}(10 \mathrm{~mL})$, and dried under reduced pressure. An analytically pure sample was obtained by recrystallization from acetone.

1-(1-Methyl-1H-pyrazol-3-yl)cyclopropanamine Dihydrochloride $(13 \mathrm{a} \cdot 2 \mathrm{HCl})$

Yield: $0.424 \mathrm{~g}$ (95\%); white solid; $\mathrm{mp} 184-186{ }^{\circ} \mathrm{C}$.

${ }^{1} \mathrm{H}$ NMR (400 MHz, DMSO- $d_{6}$ ): $\delta=8.99$ (br. s, $3 \mathrm{H}$ ), 7.68 (d, $J=2.1 \mathrm{~Hz}$, $1 \mathrm{H}), 6.25(\mathrm{~d}, J=2.1 \mathrm{~Hz}, 1 \mathrm{H}), 3.80(\mathrm{~s}, 3 \mathrm{H}), 1.49-1.34(\mathrm{~m}, 2 \mathrm{H}), 1.15-$ $1.07(\mathrm{~m}, 2 \mathrm{H}), 1 \mathrm{H}$ is exchanged with HDO.

${ }^{13} \mathrm{C}$ NMR $\left(101 \mathrm{MHz}\right.$, DMSO- $\left.d_{6}\right): \delta=149.3,132.2,101.8,38.4,31.6$, 12.8 .

MS (ESI): $m / z=138\left[\mathrm{MH}^{+}\right], 121\left[\mathrm{MH}^{+}-\mathrm{NH}_{3}\right]$.

Anal. Calcd for $\mathrm{C}_{7} \mathrm{H}_{13} \mathrm{Cl}_{2} \mathrm{~N}_{3}$ : C, 40.02; $\mathrm{H}, 6.24 ; \mathrm{N}, 20.00 ; \mathrm{Cl}, 33.75$. Found: C, 40.27; $\mathrm{H}, 6.36 ; \mathrm{N}, 19.92 ; \mathrm{Cl}, 33.70$.

1-(1-Methyl-1H-pyrazol-4-yl)cyclopropanamine Dihydrochloride $(\mathbf{1 3 b} \cdot \mathbf{2 H C l})$

Yield: $0.440 \mathrm{~g}$ (100\%); white solid; $\mathrm{mp} 170-172{ }^{\circ} \mathrm{C}$.

${ }^{1} \mathrm{H}$ NMR (400 MHz, DMSO- $d_{6}$ ): $\delta=10.30$ (br. s, $1 \mathrm{H}$ ), 9.00 (br. s, $3 \mathrm{H}$ ), $7.82(\mathrm{~s}, 1 \mathrm{H}), 7.56(\mathrm{~s}, 1 \mathrm{H}), 3.78$ (s, $3 \mathrm{H}), 1.32$ (dd, $J=6.7,5.4 \mathrm{~Hz}, 2 \mathrm{H})$, 0.96 (dd, $J=6.7,5.4 \mathrm{~Hz}, 2 \mathrm{H}$ ).

${ }^{13} \mathrm{C}$ NMR (101 MHz, DMSO- $\left.d_{6}\right): \delta=137.0,130.0,119.8,38.6,28.8$, 12.2.

MS (ESI): $m / z=138\left[\mathrm{MH}^{+}\right]$.

Anal. Calcd for $\mathrm{C}_{7} \mathrm{H}_{13} \mathrm{Cl}_{2} \mathrm{~N}_{3}$ : C, 40.02; $\mathrm{H}, 6.24 ; \mathrm{N}, 20.00 ; \mathrm{Cl}, 33.75$. Found: C, 39.94; $\mathrm{H}, 6.16 ; \mathrm{N}, 20.23 ; \mathrm{Cl}, 33.84$.

1-(1-Methyl-1H-pyrazol-5-yl)cyclopropanamine Dihydrochloride (13c.2HCl)

Yield: $0.446 \mathrm{~g}$ (100\%); white solid; mp $161-163^{\circ} \mathrm{C}$. 
${ }^{1} \mathrm{H}$ NMR (400 MHz, DMSO- $\left.d_{6}\right): \delta=9.10(\mathrm{~s}, 3 \mathrm{H}), 7.39(\mathrm{~d}, J=1.9 \mathrm{~Hz}$, $1 \mathrm{H}), 6.38$ (d, $J=1.9 \mathrm{~Hz}, 1 \mathrm{H}), 3.97(\mathrm{~s}, 3 \mathrm{H}), 1.50$ (dd, $J=7.0,5.7 \mathrm{~Hz}$, $2 \mathrm{H}), 1.15$ (dd, $J=7.0,5.7 \mathrm{~Hz}, 2 \mathrm{H}), 1 \mathrm{H}$ is exchanged with HDO.

${ }^{13} \mathrm{C}$ NMR $\left(101 \mathrm{MHz}\right.$, DMSO- $\left.d_{6}\right): \delta=138.5,137.3,108.4,37.4,26.8$, 11.1.

MS (ESI): $m / z=138\left[\mathrm{MH}^{+}\right], 121\left[\mathrm{MH}^{+}-\mathrm{NH}_{3}\right]$.

Anal. Calcd for $\mathrm{C}_{7} \mathrm{H}_{13} \mathrm{Cl}_{2} \mathrm{~N}_{3}$ : C, 40.02; $\mathrm{H}, 6.24 ; \mathrm{N}, 20.00 ; \mathrm{Cl}, 33.75$. Found: C, 39.85; H, 6.63; N, 20.31; Cl, 33.97.

\section{Preparation of Amines 14; General Procedure}

To the solution of nitrile $\mathbf{9}(0.500 \mathrm{~g}, 3.40 \mathrm{mmol}$ ) in anhydrous THF (20 $\mathrm{mL}), \mathrm{BH}_{3} \cdot \mathrm{Me}_{2} \mathrm{~S}(1.70 \mathrm{~mL}, 1.36 \mathrm{~g}, 0.0179 \mathrm{~mol})$ was added. This mixture was stirred under reflux overnight, and then cooled to r.t. $\mathrm{MeOH}(20$ $\mathrm{mL}$ ) was added and the solution was stirred at r.t. for $0.5 \mathrm{~h}$. The solvent was evaporated, and the residue was dissolved in saturated methanolic $\mathrm{HCl}(20 \mathrm{~mL})$. The mixture was heated at reflux for $1 \mathrm{~h}$ to destroy the intermediate complex. After cooling, the solvent was evaporated, $10 \%$ aq $\mathrm{NaOH}$ was added to the residue to $\mathrm{pH} 8$, and the mixture was extracted with $\mathrm{CH}_{2} \mathrm{Cl}_{2}(3 \times 20 \mathrm{~mL})$. The combined organic phases were dried over $\mathrm{Na}_{2} \mathrm{SO}_{4}$, filtered, and evaporated in vacuo to give 14. An analytical sample was obtained by column chromatography $\left(\mathrm{CHCl}_{3}-\mathrm{MeOH}-\mathrm{NEt}_{3}, 24: 1: 0.2\right)$.

\section{(1-(1-Methyl-1H-pyrazol-3-yl)cyclopropyl)methanamine (14a)}

Yield: $0.453 \mathrm{~g}$ (88\%); yellowish oil; $R_{f} 0.35\left(\mathrm{CHCl}_{3}-\mathrm{MeOH}^{-\mathrm{NEt}_{3}}\right.$, 24:1:0.2).

${ }^{1} \mathrm{H}$ NMR $\left(400 \mathrm{MHz}, \mathrm{CDCl}_{3}\right): \delta=7.19(\mathrm{~d}, J=2.1 \mathrm{~Hz}, 1 \mathrm{H}), 5.89(\mathrm{~d}, J=$ $2.1 \mathrm{~Hz}, 1 \mathrm{H}$ ), 3.78 (s, $3 \mathrm{H}$ ), 2.82 (s, $2 \mathrm{H}$ ), 2.51 (br. s, $2 \mathrm{H}$ ), 0.89-0.82 (m, $2 \mathrm{H}), 0.75-0.71(\mathrm{~m}, 2 \mathrm{H})$.

${ }^{13} \mathrm{C}$ NMR $\left(\mathrm{CDCl}_{3}, 101 \mathrm{MHz}\right): \delta=154.7,130.2,102.0,49.2,38.4,22.3$, 13.2.

MS (ESI): $m / z=152\left[\mathrm{MH}^{+}\right], 135\left[\mathrm{MH}^{+}-\mathrm{NH}_{3}\right]$.

Anal. Calcd for $\mathrm{C}_{8} \mathrm{H}_{13} \mathrm{~N}_{3}$ : C, 63.55; H, 8.67; N, 27.79. Found: C, 63.87; H, 8.42; N, 27.66.

\section{(1-(1-Methyl-1H-pyrazol-3-yl)cyclopropyl)methanamine (14b)}

Yield: $0.407 \mathrm{~g}$ (78\%); colorless oil.

${ }^{1} \mathrm{H}$ NMR $\left(400 \mathrm{MHz}, \mathrm{CDCl}_{3}\right): \delta=7.36(\mathrm{~d}, J=0.7 \mathrm{~Hz}, 1 \mathrm{H}), 7.28(\mathrm{~s}, 1 \mathrm{H})$, 3.85 (s, $3 \mathrm{H}), 2.77$ (s, $2 \mathrm{H}), 2.67$ (br. s, $2 \mathrm{H}), 0.75$ (s, $4 \mathrm{H})$.

${ }^{13} \mathrm{C}$ NMR $\left(101 \mathrm{MHz}, \mathrm{CDCl}_{3}\right): \delta=138.7,130.2,121.9,49.2,39.1,15.6$, 13.5.

MS (ESI): $m / z=152\left[\mathrm{MH}^{+}\right], 135\left[\mathrm{MH}^{+}-\mathrm{NH}_{3}\right]$.

Anal. Calcd for $\mathrm{C}_{8} \mathrm{H}_{13} \mathrm{~N}_{3}$ : C, 63.55; H, 8.67; N, 27.79. Found: C, 63.47; H, $9.04 ; \mathrm{N}, 27.43$.

\section{(1-(1-Methyl-1H-pyrazol-3-yl)cyclopropyl)methanamine (14c)}

Yield: $0.502 \mathrm{~g}$ (98\%); colorless oil.

${ }^{1} \mathrm{H} \mathrm{NMR}\left(400 \mathrm{MHz}, \mathrm{CDCl}_{3}\right): \delta=7.36(\mathrm{~d}, J=1.9 \mathrm{~Hz}, 1 \mathrm{H}), 6.08(\mathrm{~d}, J=$ $1.8 \mathrm{~Hz}, 1 \mathrm{H}$ ), 3.90 (s, $3 \mathrm{H}$ ), 2.68 (s, $2 \mathrm{H}$ ), 1.67 (br. s, 2 H), 0.85-0.82 (m, $4 \mathrm{H})$.

${ }^{13} \mathrm{C}$ NMR (101 MHz, $\left.\mathrm{CDCl}_{3}\right): \delta=143.1,137.6,107.0,49.3,36.5,20.3$, 11.0.

MS (ESI): $m / z=152\left[\mathrm{MH}^{+}\right], 135\left[\mathrm{MH}^{+}-\mathrm{NH}_{3}\right]$.

Anal. Calcd for $\mathrm{C}_{8} \mathrm{H}_{13} \mathrm{~N}_{3}$ : C, 63.55; H, 8.67; N, 27.79. Found: C, 63.86; H, 8.36; N, 27.84.

\section{Funding Information}

The work was supported by Ukrainian Government Funding (state registry No. 0114U003956) and Life Chemicals Group.

\section{Supporting Information}

Supporting information for this article is available online at https://doi.org/10.1055/s-0036-1588544.

\section{References}

(1) U. S. Food and Drug Administration. Official Website, 2015. www.fda.gov (accessed 08 Dec 2015).

(2) Peretto, I.; Radaelli, S.; Parini, C.; Zandi, M.; Raveglia, L. F.; Dondio, G.; Fontanella, L.; Misiano, P.; Bigogno, C.; Rizzi, A.; Riccardi, B.; Biscaioli, M.; Marchetti, S.; Puccini, P.; Catinella, S.; Rondelli, I.; Cenacchi, V.; Bolzoni, P. T.; Caruso, P.; Villetti, G.; Facchinetti, F.; Del, Giudice. E.; Moretto, N.; Imbimbo, B. P. J. Med. Chem. 2005, 48, 5705.

(3) Blouin, M.; Han, Y.; Burch, J.; Farand, J.; Mellon, C.; Gaudreault, M.; Wrona, M.; Lévesque, J. F.; Denis, D.; Mathieu, M. C.; Stocco, R.; Vigneault, E.; Therien, A.; Clark, P.; Rowland, S.; Xu, D.; O'Neill, G.; Ducharme, Y.; Friesen, R. J. Med. Chem. 2010, 53, 2227.

(4) Du, X.; Kim, Y. J.; Lai, S.; Chen, X.; Lizarzaburu, M.; Turcotte, S.; Fu, Z.; Liu, Q.; Zhang, Y.; Motani, A.; Oda, K.; Okuyama, R.; Nara, F.; Murakoshi, M.; Fu, A.; Reagan, J. D.; Fan, P.; Xiong, Y.; Shen, W.; Li, L.; Houze, J.; Medina, J. C. Bioorg. Med. Chem. Lett. 2012, $22,6218$.

(5) Sasmal, P. K.; Talwar, R.; Swetha, J.; Balasubrahmanyam, D.; Venkatesham, B.; Rawoof, K. A.; Neelima, Devi. B.; Jadhav, V. P.; Khan, S. K.; Mohan, P.; Srinivasa, Reddy. D.; Nyavanandi, V. K.; Nanduri, S.; Shiva, Kumar. K.; Kannan, M.; Srinivas, P.; Nadipalli, P.; Chaudhury, H.; Sebastian, V. J. Bioorg. Med. Chem. Lett. 2011, 21, 4913.

(6) Zeng, H.; Zhang, H.; Jang, F.; Zhao, L.; Zhang, J. Chem. Biol. Drug Des. 2011, 78, 333.

(7) Moss, N.; Xiong, Z.; Burke, M.; Cogan, D.; Gao, D. A.; Haverty, K.; Heim-Riether, A.; Hickey, E. R.; Nagaraja, R.; Netherton, M.; O'Shea, K.; Ramsden, P.; Schwartz, R.; Shih, D. T.; Ward, Y.; Young, E.; Zhang, Q. Bioorg. Med. Chem. Lett. 2012, 22, 7189.

(8) Meanwell, N. A. J. Med. Chem. 2011, 54, 2529.

(9) Qiao, J. X.; Cheney, D. L.; Alexander, R. S.; Smallwood, A. M.; King, S. R.; He, K.; Rendina, A. R.; Luettgen, J. M.; Knabb, R. M.; Wexler, R. R.; Lam, P. Y. Bioorg. Med. Chem. Lett. 2008, 18, 4118.

(10) (a) Papahatjis, D. P.; Nikas, S.; Tsotinis, A.; Vlachou, M.; Makriyannis, A. Chem. Lett. 2001, 3, 192. (b) Barbasiewicz, M.; Marciniak, K.; Fedoryński, M. Tetrahedron Lett. 2006, 47, 3871.

(11) (a) Langer, P.; Freiberg, W. Chem. Rev. 2004, 104, 4125. (b) Arava, V. R.; Siripalli, U. B. R.; Dubey, P. K. Tetrahedron Lett. 2005, 46, 7247. (c) Fedoryński, M.; Jonczyk, A. Org. Prep. Proced. Int. 1995, 27, 355. (d) Petrosyan, V. A.; Vasil'ev, A. A.; Tatarinova, V. I. Russ. Chem. Bull. 1994, 43, 84.

(12) Bryson, T. A.; Roth, G. A.; Jing-hau, L. Tetrahedron Lett. 1986, 27, 3685.

(13) (a) Horwell, D. C.; McKiernan, M. J.; Osborne, S. Tetrahedron Lett. 1998, 39, 8729. (b) Tsotinis, A.; Vlachou, M.; Papahatjis, D. P.; Calogeropoulou, T.; Nikas, S. P.; Garratt, P. J.; Piccio, V.; Vonhoff, S.; Davidson, K.; Teh, M. T.; Sugden, D. J. Med. Chem. 2006, 49, 3509 . 
(14) Huang, H.; Ji, X.; Wu, W.; Jiang, H. Chem. Commun. 2013, 3351.

(15) Isabel, E.; Bateman, K. P.; Chauret, N.; Cromlish, W.; Desmarais, S.; Duong, L. T.; Falgueyret, J. P.; Gauthier, J. Y.; Lamontagne, S.; Lau, C. K.; Léger, S.; LeRiche, T.; Lévesque, J.-F.; Li, C. S.; Massé, F.; McKay, D. J.; Mellon, C.; Nicoll-Griffith, D. A.; Oballa, R. M.; Percival, M. D.; Riendeau, D.; Robichaud, J.; Rodan, G. A.; Rodan, S. B.; Seto, C.; Thérien, M.; Truong, V. L.; Wesolowski, G.; Young, R. N.; Zamboni, R.; Black, W. C. Bioorg. Med. Chem. Lett. 2010, 20, 887.

(16) (a) Klapars, A.; Waldman, J. H.; Campos, K. R.; Jensen, M. S.; McLaughlin, M.; Chung, J. Y.; Cvetovich, R. J.; Chen, C. Y. J. Org. Chem. 2005, 70, 10186. (b) McCabe Dunn, J. M.; Kuethe, J. T.; Orr, R. K.; Tudge, M.; Campeau, L.-C. Org. Lett. 2014, 16, 6314. (c) Thompson, A. D.; Huestis, M. P. J. Org. Chem. 2013, 78, 762.

(17) (a) Salikov, R. F.; Platonov, D. N.; Frumkin, A. E.; Lipilin, D. L.; Tomilov, Y. V. Tetrahedron 2013, 69, 3495. (b) Furet, P.; Guagnano, V.; Fairhurst, R. A.; Imbach-Weese, P.; Bruce, I.; Knapp, M.; Fritsch, C.; Blasco, F.; Blanz, J.; Aichholz, R.; Hamon, J.; Fabbro, D.; Caravatti, G. Bioorg. Med. Chem. Lett. 2013, 23, 3741.

(18) (a) Ryabukhin, S. V.; Panov, D. M.; Granat, D. S.; Ostapchuk, E. N.; Kryvoruchko, D. V.; Grygorenko, O. O. ACS Comb. Sci. 2014, 16, 146. (b) Ivonin, S. P.; Kurpil', B. B.; Volochnyuk, D. M.; Grygorenko, O. O. Tetrahedron Lett. 2015, 56, 6248. (c) Borisov, A. V.; Voloshchuk, V. V.; Nechayev, M. A.; Grygorenko, O. O. Synthesis 2013, 45, 2413. (d) Ivonin, S. P.; Kurpil', B. B.; Bezdudny, A. V.; Volochnyuk, D. M.; Grygorenko, O. O. J. Fluorine Chem. 2015, 176, 78. (e) Zhersh, S.; Karpenko, O. V.; Ripenko, V.; Tolmachev, A. A.; Grygorenko, O. O. Cent. Eur. J. Chem. 2014, 12, 67. (f) Artamonov, O. S.; Bulda, T.; Fam, T. K.; Slobodyanyuk, E. Y.; Volochnyuk, D. M.; Grygorenko, O. O. Heterocycl. Commun. 2015, 21, 391.
(19) (a) McLellan, P.; Nelson, A. Chem. Commun. 2013, 2383. (b) Doveston, R.; Marsden, S.; Nelson, A. Drug Discovery Today 2014, 19, 813. (c) James, T.; MacLellan, P.; Burslem, G. M.; Simpson, I.; Grant, J. A.; Warriner, S. Org. Biomol. Chem. 2014, 12, 2584. (d) Foley, D. J.; Doveston, R. G.; Churcher, I.; Nelson, A.; Marsden, S. P. Chem. Commun. 2015, 11174. (e) Doveston, R. G.; Tosatti, P.; Dow, M.; Foley, D. J.; Li, H. Y.; Campbell, A. J.; House, D.; Churcher, I.; Marsden, S. P.; Nelson, A. Org. Biomol. Chem. 2015, 13, 859.

(20) Goldberg, F. W.; Kettle, J. G.; Kogej, T.; Perry, M. W. D.; Tomkinson, N. P. Drug Discovery Today 2015, 20, 11.

(21) Nadin, A.; Hattotuwagama, C.; Churcher, I. Angew. Chem. Int. Ed. 2012, 51, 1114 .

(22) (a) Nosik, P. S.; Ryabukhin, S. V.; Artamonov, O. S.; Grygorenko, O. O. Monatsh. Chem. 2016, 147, 1629. (b) Azami, H.; Barrett, D.; Tanaka, A.; Sasaki, H.; Matsuda, K.; Sakurai, M.; Terasawa, T.; Shirai, F.; Chiba, T.; Matsumoto, Y.; Tawara, S. Bioorg. Med. Chem. 2001, 9, 961.

(23) Labroli, M.; Paruch, K.; Dwyer, M. P.; Alvarez, C.; Keertikar, K.; Poker, C.; Rossman, R.; Duca, J. S.; Fischmann, T. O.; Madison, V.; Parry, D.; Davis, N.; Seghezzi, W.; Wiswell, D.; Guzi, T. J. Bioorg. Med. Chem. Lett. 2011, 21, 471.

(24) Instant JChem was used for prediction of the physicochemical properties of the compounds, Instant JChem version 17.2.13.0, 2017, ChemAxon ( http://www.chemaxon.com).

(25) Kombarov, R.; Altieri, A.; Genis, D.; Kirpichenok, M.; Kochubey, V.; Rakitina, N.; Titarenko, Z. Mol. Diversity 2010, 14, 193.

(26) Armarego, W. L. F.; Chai, C. L. L. Purification of Laboratory Chemicals; Elsevier: Oxford, 2003. 